\section{STRAIGHT LINE VS TRUE DISTANCE TO NEAREST AED - DOES IT MATTER FOR CARDIAC ARREST COVERAGE?}

LI M Karlsson*, ${ }^{1} \mathrm{~KB}$ Sondergaard, ${ }^{1} \mathrm{CM}$ Hansen, ${ }^{1} \mathrm{M}$ Wissenberg, ${ }^{2} \mathrm{SM}$ Hansen, ${ }^{3} \mathrm{FK}$ Lippert, ${ }^{1} \mathrm{~S}$ Rajan, ${ }^{2} \mathrm{~K}$ Kragholm, ${ }^{2} \mathrm{~S}$ Møller, ${ }^{1,4} \mathrm{GH}$ Gislason, ${ }^{2} \mathrm{C}$ Torp-Pedersen, ${ }^{1,3} \mathrm{~F}$ Folke. ${ }^{1}$ Department of Cardiology, Copenhagen University Hospital Gentofte, Denmark; ${ }^{2}$ Departments of Clinical Epidemiology and Cardiology, Aalborg University Hospital, Denmark; ${ }^{3}$ Emergency Medical Services Copenhagen, the Capital Region of Denmark;

${ }^{4}$ National Institute of Public Health, University of Southern Denmark, Copenhagen

10.1136/bmjopen-2017-EMSabstracts.42

Aim International guidelines recommend automated external defibrillators (AEDs) to be deployed within 1_ min brisk walk distance to high-risk out-of-hospital cardiac arrest (OHCA) locations. Most studies translate this to a straight line distance of $100 \mathrm{~m}$ while the true distance may be longer. We aimed to investigate how straight line versus true distances affected the AED coverage of nearby OHCA.

Methods We identified all OHCAs (2002-2014) and all public available AEDs in 2014, in Copenhagen, Denmark. Each arrest and AED was geocoded and straight line and true distance from arrest to the nearest AED was calculated. An OHCA was defined as covered by an AED if $\leq 100 \mathrm{~m}$.

Results Of 4507 OHCAs, 22.3\% occurred in public $(n=1003)$ and $77.7 \%(n=3504)$ in residential locations. In 2014, there were 1134 public available AEDs. In total, the median distance in straight line was $148 \mathrm{~m}$ (interquartile range (IQR):87226), versus $224 \mathrm{~m}$ (IQR:128-343) in true distance, with an AED coverage of $30.9 \% \quad(n=1394)$, versus $18.5 \% \quad(n=832)$, respectively. For public arrests, the median distance and AED coverage in straight line was $107 \mathrm{~m}$ (IQR:53-185) and $48.1 \%$ $(\mathrm{n}=482)$, versus $167 \mathrm{~m}$ (IQR:70-274) and 33.6\% $(\mathrm{n}=337)$ in true distances. The corresponding numbers for residential OHCAs were 158m (IQR:98-239) and 26.0\% ( $n=912)$ for straight line distance, versus 241m (IQR:146-354) and 14.1\% $(\mathrm{n}=495)$ for true distance.

Conclusion Using true instead of straight line distance provides a more realistic estimation of AED coverage and could help improve public access defibrillation programs.

Conflict of interest None declared.

Funding Dr. L. I. M. Karlsson is supported by a fund from The Danish foundation TrygFonden, who has no influence on study design; in the collection, analysis, or interpretation of data.

\section{TIME DELAY TO CONTACT THE MEDICAL DISPATCH - EUROCALL STUDY IN ROMANIA}

${ }^{1} \mathrm{D}$ Cimpoesu*, ${ }^{1} \mathrm{O}$ Popa, ${ }^{2} \mathrm{Gr}$. T Popa. ${ }^{1}$ University of Medicine and Pharmacy; ${ }^{2}$ lasi Paul Nedelea-Emergency Department Emergency County Hospital Sf.Spiridon, lasi

\subsection{6/bmjopen-2017-EMSabstracts.43}

Aim To assess the time to connect the telephone call to emergency medical dispatch.

Methods Regional randomised study as a part of a prospective, multicenter study was conducted in April 2013 within the Medical Dispatch, Iasi, Romania. The distribution of the 112 calls followed a diurnal distribution using a computer generated sets of random numbers. We analysed the time to contact medical dispatch and its components (Time to first beep, First beep to 112 operator answer,First beep to medical dispatch answer).
Results There were planned to do 180 calls (6 calls/24 hour) and we performed only 129 calls (71,66\%). The average "Time to first beep" was $3.515 \pm 3.447 \mathrm{~s}$, "First beep to 112 operator answer" was $6.823 \pm 5.559 \mathrm{~s}$ and "First beep to medical dispatch answer" was $24.259 \pm 19.584$ s. No statistically significant difference in terms of response time when using mobile or landline phone. We found an negative correlation between "First beep to medical dispatch answer" and the hour of calls $(\mathrm{p}<0,05)$; thus, the average time of this interval between $00.00-06.00$ hour was of $41.724 \pm 36105 \mathrm{~s}$.

Conclusion There is a great variation regarding the time to contact the medical dispatcher, mainly during the night. This could be an additional time with negative influence for out of hospital cardiac arrest outcomes.

\section{REFERENCE}

1. Nikolaou N, Castrén M, Monsieurs KG, Cimpoesu D, Georgiou M, Raffay V, Koster $R$, Hunyadi-Anticevi $S$, Truhlár A, Bossaert L, The EUROCALL investigators. Time delays to reach dispatch centres in different regions in Europe. Are we losing the window of opportunity? - The EUROCALL study. Resuscitation 2017:111:8-13.

Conflict of interest None declared. Funding None declared.

\section{POINT-OF-CARE ANALYSIS OF LACTATE FROM INTRAOSSEOUS SAMPLES DURING RESUSCITATION}

${ }^{1,2} \mathrm{M}$ Jousi*, ${ }^{3} \mathrm{~A}$ Nelskylä, ${ }^{3} \mathrm{M}$ Skrifvars, ${ }^{4} \mathrm{G}$ Ristagno, ${ }^{2} \mathrm{~J}$ Nurmi. ${ }^{1}$ FinnHEMS Research and Development Unit, Finland; ${ }^{2}$ Department of Emergency Medicine and Services, Helsinki University and Helsinki University Hospital, Finland; ${ }^{3}$ Department of Anaesthesiology, Intensive Care and Pain Medicine, Helsinki University and Helsinki University Hospital, Finland; ${ }^{4}$ Istituto di Ricerche Farmacologiche "Mario Negri", Milano, Italy

\subsection{6/bmjopen-2017-EMSabstracts.44}

Aim Intraosseous (IO) access with power-driven devices has become a commonly used method of vascular access during cardiopulmonary resuscitation (CPR). Blood aspirated to confirm correct IO needle position could readily be available for point-of-care (POC) testing. The aim was to investigate how POC lactate levels of intraosseous blood reflect the lactate values in systemic circulation during VF and resuscitation in order to see whether POC IO samples could be used for clinical decision-making during CPR.

Methods We conducted an experimental study comparing POC results of lactate from intraosseous, arterial and venous blood of 23 piglets undergoing induced cardiac arrest (VF) and CPR. All blood samples were analysed with i-STAT POC device and the results were compared using Bland-Altman method (ref 1).

Results Prior to VF the IO lactate levels were similar to arterial and venous samples (bias $[95 \% \mathrm{CI}]$ between $\mathrm{IO}$ and arterial samples was $0.11 \mathrm{mmol} / \mathrm{L}[-0.02-0.24]$ and between $\mathrm{IO}$ and venous samples $0.03 \mathrm{mmol} / \mathrm{L}[-0.25-0.31])$. Five minutes after onset of VF, intraosseous lactate levels had increased more than arterial and venous values (bias $3.76 \mathrm{mmol} / \mathrm{L}$ [1.93-5.59] and $3.52 \mathrm{mmol} / \mathrm{L}$ [1.41-5.64] respectively). Five minutes after initiation of CPR with an automatic CPR device (LucasTM) the difference diminished (bias $0.81 \mathrm{mmol} / \mathrm{L}$, [-0.31-1.93] and $1.50 \mathrm{mmol} / \mathrm{L}$ [0.07-2.92]).

Conclusion Intraosseous lactate values showed good agreement with arterial and venous values before cardiac arrest, but IO values were clearly higher during VF and CPR. During 